\title{
PARTIAL HOMOMORPHIC IMAGES OF BRANDT GROUPOIDS ${ }^{1}$
}

\author{
A. H. CLIFFORD
}

The main purpose of the present note is to show (Theorem 2) that any regular $D$-class of any semigroup is a partial homomorphic image of a Brandt groupoid. It follows from this that a semigroup with zero is a partial homomorphic image of a Brandt semigroup if and only if it is regular and 0-bisimple.

In the first section, an alternative formulation is given of the determination by H.-J. Hoehnke [1] of all partial homomorphisms of a Brandt groupoid into an arbitrary semigroup. This is first done (Theorem 1) for any completely 0 -simple semigroup. The result is a straightforward generalization of Theorem 3.14 of [2], in which all partial homomorphisms of one completely 0 -simple semigroup into another are determined. The present terminology is that of [2]; Hoehnke omits the adjective "partial." Basic definitions given in [2] will not be repeated here; likewise, references to the fundamental work of Brandt, Rees, Green, and Munn can be found in [2].

1. Partial homomorphisms of a completely 0 -simple semigroup. Let $S$ and $S^{*}$ be semigroups with zero elements 0 and $0^{*}$, respectively. A mapping $\theta$ of $S$ into $S^{*}$ is called a partial homomorphism if (i) $0 \theta=0^{*}$, and (ii) $(a b) \theta=(a \theta)(b \theta)$ for every pair of elements $a, b$ of $S$ such that $a b \neq 0$. The restriction of $\theta$ to $S \backslash 0$ is then a partial homomorphism of the partial groupoid $S \backslash 0$ into $S^{*}$ as defined in [2, p. 93]. By agreeing to the trivial convention (i), there is no essential distinction between partial homomorphisms of $S$ into $S^{*}$ and of $S \backslash 0$ into $S^{*}$. Moreover, we need not require that $S^{*}$ have a zero element; if it does not, we adjoin a zero element $0^{*}$ to it for the application of (i).

The author's interest in partial homomorphisms originated in the fact that they arise naturally in the theory of extensions of semigroups $[2, \S 4.4]$.

A partial homomorphism $\theta: S \rightarrow S^{*}$ evidently preserves regularity $[2$, p. 26] and Green's relations $R, \mathcal{L}, D$, and $\mathcal{H}$ [2, p. 47]. It follows that if $S$ is regular and 0 -bisimple (i.e., $S \backslash 0$ is a D-class of $S[2$, p. 76]), then $(S \backslash 0) \theta$ is contained in a regular D-class $D$ of $S^{*}$. This is the case, in particular, if $S$ is completely 0 -simple [2, Theorem

Received by the editors December 30, 1963.

1 This work was supported by the National Science Foundation grant GP 1791. 
2.51, p. 79]. Since a Brandt semigroup $B^{0}$ is just a completely 0 -simple inverse semigroup [2, Theorem 3.9, p. 102], we conclude, finally, that if $\theta$ is a partial homomorphism of a Brandt groupoid $B=B^{0} \backslash 0$ into a semigroup $S^{*}$, then $B \theta$ is contained in a regular D-class $D$ of $S$. One might think that these successive particularizations would result in some restriction on $D$, particularly if $\theta$ is onto; the object of this note is to show that this is not the case (Theorem 2 below).

Let $D$ be a regular D-class of $S^{*}$. Let

$$
\left\{R_{i^{*}}: i^{*} \in I^{*}\right\} \quad \text { and } \quad\left\{L_{\lambda^{*}}: \lambda^{*} \in \Lambda^{*}\right\}
$$

be the $R$-classes and $\&$-classes, respectively, of $S^{*}$ contained in $D$. Then $H_{i^{*} \lambda^{*}}=R_{i^{*}} \cap L_{\lambda^{*}}$ are the $\mathcal{F}$-classes of $S^{*}$ contained in $D$. We know that at least one of these must contain an idempotent, and so be a maximal subgroup of $S^{*}[2$, Theorem 2.16, p. 59]; choose one such and call it $H^{*}=H_{1^{*} 1^{*}}, 1^{*}$ being an element of $I^{*} \cap \Lambda^{*}$. For each $i^{*}$ in $I^{*}$, pick $r_{i^{*}}$ in $H_{i^{* 1 *}}$, and for each $\lambda^{*}$ in $\Lambda^{*}$ pick $q_{\lambda^{*}}$ in $H_{1^{*} \lambda^{*}}$. Then [2, Theorem 3.4 , p. 92], every element of $D$ is uniquely representable in the form

$$
r_{i^{*}} x q_{\lambda^{*}} \quad\left(x \in H^{*} ; i^{*} \in I^{*}, \lambda^{*} \in \Lambda^{*}\right) .
$$

We regard the triple $\left(x ; i^{*}, \lambda^{*}\right)$ as coordinates of the element (1).

By the Rees Theorem [2, Theorem 3.5, p. 94], a completely 0simple semigroup can be represented as a regular Rees $I \times \Lambda$ matrix semigroup $\mathfrak{T}^{0}(G ; I, \Lambda ; P)$ over a group with zero $G^{0}$, and with $\Lambda \times I$ sandwich matrix $P=\left(p_{\lambda i}\right)$. The elements of $\mathfrak{T}^{0}$ can be represented as triples $(a ; i, \lambda)$ multiplying according to

$$
\text { (2) }(a ; i, \lambda)(b ; j, \mu)=\left(a p_{\lambda j} b ; i, \mu\right) \quad\left(a, b \in G^{0} ; i, j \in I ; \lambda, \mu \in \Lambda\right) \text {. }
$$

In fact, the proof of the Rees Theorem amounts to coordinatizing the D-class $\mathfrak{T}^{0} \backslash 0$. It should be remarked that, for an arbitrary regular D-class $D$, the elements (1) do not have a simple law of multiplication like (2).

THEOREM 1. Let $S$ be a completely 0 -simple semigroup represented as a regular Rees $I \times \Lambda$ matrix semigroup $\mathfrak{T}^{0}(G ; I, \Lambda ; P)$. Let $\theta$ be a partial homomorphism of $S$ into a semigroup $S^{*}$. Then $(S \backslash 0) \theta$ is contained in a regular D-class $D$ of $S$. Let $D$ be coordinatized as in (1). Then

$$
(a ; i, \lambda) \theta=r_{i \phi} u_{i}(a \omega) v_{\lambda} q_{\lambda \psi} \quad(a \in G ; i \in I, \lambda \in \Lambda),
$$

where (i) $\phi: I \rightarrow I^{*}$ and $\psi: \Lambda \rightarrow \Lambda^{*}$ are mappings such that if $p_{\lambda_{i}} \neq 0$ then $q_{\lambda \psi} r_{i \phi} \in H^{*}$;

(ii) $\omega: G \rightarrow H^{*}$ is a (group) homomorphism; 
(iii) $u: I \rightarrow H^{*}$ and $v: \Lambda \rightarrow H^{*}$ are mappings such that if $p_{\lambda_{i}} \neq 0$ then

$$
p_{\lambda i} \omega=v_{\lambda}\left(q_{\lambda \psi} r_{i \phi}\right) u_{i}
$$

The mappings $\phi, \psi, \omega, u, v$ are uniquely determined by $\theta$. Conversely, if mappings $\phi, \psi, \omega, u, v$ are given satisfying (i), (ii), and (iii), then (3) defines a partial homomorphism $\theta$ of $S \backslash 0$ into $D$.

Proof. The proof is so much like that of Theorem 3.14 of $[2, \mathrm{p}$. 109], that we give only the outline. We can assume that the entry $p_{11}$ of $P$ is not zero. The mappings $\phi$ and $\psi$ are determined by

$$
R_{i} \theta \subseteq R_{i \phi}, \quad L_{\lambda} \theta \subseteq L_{\lambda \psi}
$$

where $\left\{R_{i}: i \in I\right\}$ are the $R$-classes, and $\left\{L_{\lambda}: \lambda \in \Lambda\right\}$ are the $\&$ classes, of $S$. This implies that

$$
(a ; i, \lambda) \theta=r_{i \phi} x q_{\lambda \psi}
$$

for some $x$ in $H^{*}$. If $p_{\lambda i} \neq 0$, then $\left(p_{\lambda i}^{-1} ; i, \lambda\right) \theta$ is an idempotent in $H_{i \phi, \lambda \psi}$, and it follows that $q_{\lambda \psi} r_{i \phi} \in H^{*}[2$, Theorem 2.17, p. 59]. Defining $\omega: G \rightarrow H^{*}$ by

$$
\left(p_{11}^{-1}, a ; 1,1\right) \theta=r_{1 \phi} h_{0}^{-1}(a \omega) q_{1 \psi} \quad\left(h_{0}=q_{1 \psi} r_{1 \phi}\right),
$$

a brief calculation, using the uniqueness of the representation (1), shows that $\omega$ is a homomorphism. For each $i \in I$ and $\lambda \in \Lambda$ we define $u_{i}$ and $v_{\lambda}$ in $H^{*}$ by

$$
\begin{gathered}
(e ; i, 1) \theta=r_{i \phi} u_{i} q_{1 \psi}, \\
\left(p_{11}^{-1} 1, \lambda\right) \theta=r_{1 \phi} h_{0}^{-1} v_{\lambda} q_{\lambda \psi} .
\end{gathered}
$$

Applying $\theta$ to

$$
(a ; i, \lambda)=(e ; i, 1)\left(p_{11}^{-1} a ; 1,1\right)\left(p_{11}^{-1} ; 1, \lambda\right),
$$

we obtain (3). Applying $\theta$ to (2) and using (3), again with the uniqueness of (1), we obtain (4). This last step can be inverted to yield the converse part of the theorem.

From a constructive point of view, Theorem 1 has the drawback that, for given $\phi, \psi$, and $\omega$ satisfying (i) and (ii), there is no assurance that $u$ and $v$ can be found so as to satisfy (iii). This drawback disappears, however, when $S$ is a Brandt semigroup $B^{0}$. Here we can assume $B^{0}=\mathscr{N}^{0}(G ; I, I ; \Delta)$, where $\Delta=\left(\delta_{i j}\right)$ is the $I \times I$ identity matrix over $G^{0}[2$, Theorem 3.9, p. 102]. The condition (4) now reduces to

$$
e^{*}=v_{i} q_{i \psi} r_{i \phi} u_{i} \quad(\text { all } i \in I),
$$


where $e^{*}$ is the identity element of $H^{*}$; or, what is equivalent, to

$$
v_{i}=u_{i}^{-1}\left(q_{i \psi} r_{i \phi}\right)^{-1} \text {. }
$$

We note that $q_{i \psi} r_{i \phi} \in H^{*}$ by (i). Thus we can always satisfy (iii) by choosing $u: I \rightarrow H^{*}$ arbitrarily, and then defining $v: I \rightarrow H^{*}$ by (5). Formula (3) becomes

$$
(a ; i, j) \theta=r_{i \phi} u_{i}(a \omega) u_{j}^{-1}\left(q_{j \psi} r_{j \phi}\right)^{-1} q_{j \psi} .
$$

This differs from Hoehnke's formula (16) of [1, Part III, p. 97], chiefly because a definite coordinate system has been chosen for $D$, independent of $\theta$.

Now let $D$ itself be a Brandt groupoid, say

$$
D=B^{*}=\mathfrak{N}^{0}\left(H^{*} ; I^{*}, I^{*} ; \Delta^{*}\right) \backslash 0 .
$$

Let us use square brackets to represent the elements $\left[x^{*} ; i^{*}, j^{*}\right]$ of $B^{*}$. It is natural to choose $r_{i^{*}}=\left[e^{*} ; i^{*}, 1^{*}\right]$ and $q_{i^{*}}=\left[e^{*} ; 1^{*}, i^{*}\right]$. We then have $q_{i^{*}} r_{i^{*}}=\left[e^{*} ; 1^{*}, 1^{*}\right]$, while $q_{i^{*}} r_{j^{*}}=0$ in $B^{* 0}$, or is undefined in $B^{*}$, if $i^{*} \neq j^{*}$. Hence condition (i) of Theorem 1 requires that $i \psi=i \phi$ for every $i$ in $I$; that is, $\psi=\phi$. (5) becomes simply $v_{i}=u_{i}^{-1}$, and (6) becomes

$$
(a ; i, j) \theta=\left[u_{i}(a \omega) u_{j}^{-1} ; i \phi, j \phi\right] .
$$

Thus every partial homomorphism $\theta$ of one Brandt groupoid, $B$, into another, $B^{*}$, is given by (7) in terms of (i) an arbitrary mapping $\phi: I \rightarrow I^{*}$, (ii) an arbitrary homomorphism $\omega: G \rightarrow H^{*}$, and (iii) an arbitrary mapping $u: I \rightarrow H^{*}$. (7) is equivalent to Hoehnke's formula (22) in [1, Part I, p. 164]. It can also be obtained by specialization from Theorem 3.14 of [2].

2. Partial homomorphic images of Brandt groupoids. We come now to the main result of the present note.

THEOREM 2. Any regular D-class of any semigroup is a partial homomorphic image of some Brandt groupoid.

Proof. Let $D$ be a regular $D$-class of a semigroup $S$. Let

$$
\left\{R_{i}: i \in I\right\} \text { and }\left\{L_{\lambda}: \lambda \in \Lambda\right\}
$$

be the $R$-classes and $\mathcal{L}$-classes, respectively, of $S$ contained in $D$. As usual, we may assume that $I$ and $\Lambda$ have an element 1 in common such that $H_{11}=R_{1} \cap L_{1}$ is a group. But now we shall also assume, as we evidently may, that $I$ and $\Lambda$ are otherwise disjoint: $I \cap \Lambda=\{1\}$.

As usual, choose $r_{i}$ in $H_{i 1}$ and $q_{\lambda}$ in $H_{1 \lambda}$ in any way, for $i$ in $I \backslash 1$ and $\lambda$ in $\Lambda \backslash 1$, and choose $r_{1}=q_{1}=e_{11}$, the identity element of $H_{11}$. As 
in (1), without the stars, every element of $D$ is uniquely representable in the form

$$
r_{i} a q_{\lambda} \quad\left(a \in H_{11} ; i \in I, \lambda \in \Lambda\right) .
$$

For $i$ in $I \backslash 1$ and $\lambda$ in $\Lambda \backslash 1$, let $q_{i}$ be any inverse of $r_{i}$ in $R_{1}$, and let $r_{\lambda}$ be any inverse of $q_{\lambda}$ in $L_{1}$. Then

$$
q_{\alpha} r_{\alpha}=e_{11} \quad(\text { all } \alpha \text { in } I \cup \Lambda) .
$$

Let $B=\operatorname{M}^{0}\left(H_{11} ; I \cup \Lambda, I \cup \Lambda ; \Delta\right) \backslash 0$. Denote the elements of $B$ by triples $(a ; \alpha, \beta)$. Multiplication in $B$ is given by

$$
(a ; \alpha, \beta)(b ; \beta, \gamma)=(a b ; \alpha, \gamma) \quad\left(a, b \in H_{11} ; \alpha, \beta, \gamma \in I \cup \Lambda\right)
$$

Products $(a ; \alpha, \beta)\left(b ; \beta^{\prime}, \gamma\right)$ with $\beta \neq \beta^{\prime}$ are not defined in $B$ (and are zero in $B^{0}$ ). Define $\theta: B \rightarrow D$ as follows:

$$
(a ; \alpha, \beta) \theta=r_{\alpha} a q_{\beta} \quad\left(a \in H_{11} ; \alpha, \beta \in I \cup \Lambda\right) .
$$

Then, because of (9),

$$
\begin{aligned}
(a ; \alpha, \beta) \theta(b ; \beta, \gamma) \theta & =r_{\alpha} a q_{\beta} r_{\beta} b q_{\gamma}=r_{\alpha} a b q_{\gamma} \\
& =(a b ; \alpha, \gamma) \theta .
\end{aligned}
$$

From this and (10), it follows that $\theta$ is a partial homomorphism of $B$ into $D$. Moreover, $B \theta=D$, since $B \theta$ contains all the elements $r_{i} a q_{\lambda}$ of (8).

As described in $\$ 3.3$ of [2], if we adjoin a zero element 0 to a Brandt groupoid $B$, defining $a b=0$ if $a b$ is undefined in $B$, we obtain a Brandt semigroup $B^{0}$, that is, a completely 0 -simple inverse semigroup. The following is immediate from Theorem 2 and the first assertion in Theorem 1.

CoROllary 1. A semigroup with zero is a partial homomorphic image of some Brandt semigroup if and only if it is regular and 0-bisimple.

As defined in [2, p. 93], a partial isomorphism is a partial homomorphism which is one-to-one and onto. Not every regular D-class is a partial isomorphic image of some Brandt groupoid, and the question of telling which ones are remains unsettled. The next theorem gives a sufficient condition.

Theorem 3. Let $D$ be a regular D-class of a semigroup $S$ with the property that it is possible to set up a one-to-one correspondence between the $R$-classes $R$ of $D$ and the $\mathcal{L}$-classes $\mathfrak{L}$ of $D$ such that if $R$ and $L$ correspond, then $R \cap L$ contains an idempotent. Then $D$ is a partial isomorphic image of the Brandt groupoid having the same structure group as $D$ and the same number of R-classes (and $\mathfrak{L}$-classes) as $D$. 
Proof. By hypothesis, we can index the $R$-classes and the $\mathcal{L}$-classes of $D$ by the same index set $I$, such that for each $i$ in $I, R_{i} \cap L_{i}$ contains an idempotent $e_{i}$. The $\mathcal{F}$-class $H_{i i}=R_{i} \cap L_{i}$ is then the maximal subgroup $H_{e_{i}}$ of $S$ containing $e_{i}$. Let $1 \in I$, and pick $q_{i}$ in $H_{1 i}$ in any way for $i$ in $I \backslash 1$, and let $q_{1}=e_{1}$. Let $q_{i}^{\prime}$ be the inverse of $q_{i}$ in $H_{i 1}$; such exists since both $H_{11}$ and $H_{i i}$ contain idempotents [2, Theorem 2.18 , p. 60]. Take $B=\mathfrak{T C}^{0}\left(H_{11} ; I, I ; \Delta\right) \backslash 0$ and define $\theta: B \rightarrow D$ by

$$
(a ; i, j) \theta=q_{i}^{\prime} a q_{j} \quad\left(a \in H_{11} ; i, j \in I\right) .
$$

Since every element of $D$ is uniquely expressible in the form on the right-hand side of (11), and $q_{j} q_{j}^{\prime}=e_{1}$, we see at once that $\theta$ is a partial isomorphism of $B$ onto $D$.

$B$ is unique, to within isomorphism, since any Brandt groupoid is completely determined by its structure group and the cardinal number of its $R$-classes (or $\mathscr{L}$-classes).

COROLlaRY 2. Every 0-bisimple inverse semigroup $S$ is a partial isomorphic image of the Brandt semigroup having the same structure group as $S$ and the same number of idempotents as $S$.

Proof. The hypothesis of Theorem 3 is satisfied by any inverse semigroup [2, Corollary 2.19, p. 60]. For 0-bisimple inverse semigroups, in particular, for Brandt semigroups, the sets of $R$-classes, $\mathcal{L}$-classes, and nonzero idempotents all have the same cardinal.

We conclude with an example to show that a regular 0-bisimple semigroup may be a partial isomorphic image of a Brandt semigroup, but not of one having the same structure group.

Let $B=\pi^{\circ}(E ; I, I ; \Delta) \backslash 0$, where $E=\{e\}$ is a one-element group, and $I=\{1,2\}$. Let $S \backslash 0=H \times E$, where $H$ is a cyclic group $\{e, a\}$ of order 2, and $E$ is a right zero semigroup of order 2 . We may represent the elements of $S$ as pairs $(x ; i)$ with $x \in H, i \in I$, multiplying as follows:

$$
(x ; i)(y ; j)=(x y ; j) \quad(x, y \in H ; i, j \in I) .
$$

Define $\theta: B^{0} \rightarrow S$ by

$$
\begin{array}{ll}
(e ; 1,1) \theta=(e ; 1), & (e ; 1,2) \theta=(a ; 2) \\
(e ; 2,1) \theta=(a ; 1), & (e ; 2,2) \theta=(e ; 2)
\end{array}
$$

and $0 \theta=0$. Clearly $\theta$ is one-to-one and onto, and it is easy to verify that it is a partial homomorphism.

On the other hand, $S$ cannot be a partial isomorphic irnage of any Brandt semigroup $B^{0}$ having structure group of order 2. For $B$ must then have order twice a square, whereas $S \backslash 0$ has order 4. 


\section{REFERENCES}

1. H.-J. Hoehnke, Zur Theorie der Gruppoide. I, Math. Nachr. 24 (1962), 137168; III, Acta Math. 13 (1962), 91-100.

2. A. H. Clifford and G. B. Preston, The algebraic theory of semigroups, Math. Surveys No. 7, Amer. Math. Soc., Providence, R. I. 1961.

TUlane UNIVERSITY

\section{EVERY STANDARD CONSTRUCTION IS INDUCED BY A PAIR OF ADJOINT FUNCTORS}

H. KLEISLI

In this note, we prove the converse of the following result of P. Huber [2]. Let $F: \mathfrak{K} \rightarrow \mathscr{L}$ and $G: \mathfrak{L} \rightarrow \mathcal{K}$ be covariant adjoint functors, that is, functors such that there exist two (functor) morphisms $\zeta: I \rightarrow G F$ and $\eta: F G \rightarrow I$ satisfying the relations

$$
\begin{aligned}
& (\eta * F) \circ(F * \zeta)=\imath * F \\
& (G * \eta) \circ(\zeta * G)=\imath * G .
\end{aligned}
$$

Then, the triple $(C, k, p)$ given by

$$
C=F G, \quad k=\eta \quad \text { and } \quad p=F * \zeta * G
$$

is a standard construction in $\mathcal{L}$, that is, $C$ is a covariant functor, $k: C \rightarrow I$ and $p: C \rightarrow C^{2}$ are (functor) morphisms, and the following relations hold:

$$
\begin{aligned}
& (k * C) \circ p=(C * k) \circ p=\imath * C, \\
& (p * C) \circ p=(C * p) \circ p .
\end{aligned}
$$

This standard construction is said to be induced by the pair of adjoint functors $F$ and $G$. For further explanation of the notation and terminology, see [2], or the appendix of [1].

TheOREM. Let $(C, k, p)$ be a standard construction in a category $\mathfrak{L}$. Then there exists a category $\Re$ and two covariant functors $F: \mathfrak{K} \rightarrow \mathfrak{L}$ and $G: \mathfrak{L} \rightarrow \mathfrak{K}$ such that

(i) $F$ is (left) adjoint to $G$,

(ii) $(C, k, p)$ is induced by $F$ and $G$.

Received by the editors March 2, 1964. 